\title{
Some remarks on rearrangement for nonlocal functionals
}

\author{
Hoai-Minh Nguyen ${ }^{\mathrm{a}}$, Marco Squassina ${ }^{\mathrm{b}, *, 1}$ \\ a Department of Mathematics, EPFL SB CAMA, Station 8 CH-1015 Lausanne, Switzerland \\ b Dipartimento di Matematica e Fisica, Università Cattolica del Sacro Cuore, Via dei Musei 41 , I-25121 \\ Brescia, Italy
}

\section{A R T I C L E I N F O}

\section{Article history:}

Received 26 April 2017

Accepted 16 June 2017

Communicated by Enzo Mitidieri

MSC 2010:

$46 \mathrm{E} 35$

$28 \mathrm{D} 20$

$82 \mathrm{~B} 10$

49A50

Keywords:

Polarization

Nonlocal functionals

Characterization of Sobolev spaces

\section{A B S T R A C T}

We prove that a nonlocal functional approximating the standard Dirichlet $p$-norm fails to decrease under two-point rearrangement. Furthermore, we get other properties related to this functional such as decay and compactness.

๑) 2017 Elsevier Ltd. All rights reserved.

\section{Introduction}

The well-known Polya-Szegö inequality $[15,29,30]$ states that if $u \in W^{1, p}\left(\mathbb{R}^{N}, \mathbb{R}^{+}\right)$then

$$
\int_{\mathbb{R}^{N}}\left|\nabla u^{*}\right|^{p} d x \leq \int_{\mathbb{R}^{N}}|\nabla u|^{p} d x
$$

where $u^{*}$ is the Schwarz symmetric rearrangement of $u$. This inequality has relevant applications in the study of isoperimetric inequalities, in the Faber-Krahn inequality and in the determination of optimal constants in the Sobolev inequality $[2,36]$. This kind of inequalities still holds in the nonlocal case, e.g. for the standard fractional norm, namely for $u \in W^{s, p}\left(\mathbb{R}^{N}, \mathbb{R}^{+}\right)$

$$
\iint_{\mathbb{R}^{2 N}} \frac{\left|u^{*}(x)-u^{*}(y)\right|^{p}}{|x-y|^{N+p s}} d x d y \leq \iint_{\mathbb{R}^{2 N}} \frac{|u(x)-u(y)|^{p}}{|x-y|^{N+p s}} d x d y,
$$

\footnotetext{
* Corresponding author.

E-mail addresses: hoai-minh.nguyen@epfl.ch (H.-M. Nguyen), marco.squassina@unicatt.it (M. Squassina).

${ }^{1}$ The second author is member of Gruppo Nazionale per l'Analisi Matematica, la Probabilità e le loro Applicazioni (GNAMPA) of the Istituto Nazionale di Alta Matematica (INdAM).
} 
for $p \geq 1$ and $s \in(0,1)$, see e.g. [1,3]. Actually this inequality implies (1.1) by a straightforward application of a result by Bourgain, Brezis and Mironescu $[4,5]$ which confirms that

$$
\lim _{s \nearrow 1}(1-s) \iint_{\mathbb{R}^{2 N}} \frac{|v(x)-v(y)|^{p}}{|x-y|^{N+p s}} d x d y=K_{N, p} \int_{\mathbb{R}^{N}}|\nabla v|^{p}, \quad \text { for } v \in W^{1, p}\left(\mathbb{R}^{N}\right),
$$

where

$$
K_{N, p}:=\int_{\mathbb{S}^{N-1}}|\boldsymbol{e} \cdot \sigma|^{p} d \sigma, \quad \text { for some } \boldsymbol{e} \in \mathbb{S}^{N-1}, \text { the unit sphere in } \mathbb{R}^{N} \text {. }
$$

Polarization by closed half spaces $H \subset \mathbb{R}^{N}$ containing the origin is an elementary form of symmetrization and it is a key tool in order to investigate various rearrangements inequalities. The polarization $u^{H}$ with respect to $H$, see the definition (2.1), also called two-point rearrangement, essentially compares the values of $u$ on the two sides of $\partial H$ and keeps the largest values inside $H$ and the smallest values outside $H$, cf. [14,16-18]. Since the first achievements obtained in [14], the approximation in $L^{p}\left(\mathbb{R}^{N}\right)$ of $u^{*}$ via iterated polarizations of $u$ has been refined in various ways. It is now known that there exists an explicit and universal (i.e. independent of $u$ ) sequence of closed half spaces $\left\{H_{n}\right\}_{n \in \mathbb{N}}$ of $\mathbb{R}^{N}$ containing the origin such that a suitable sequence of iterated polarizations of $u$ with respect to $H_{n}$ strongly converges to $u^{*}$ in $L^{p}\left(\mathbb{R}^{N}\right)$, see [38] and the references therein. It is thus natural to derive the rearrangement inequalities (1.1)-(1.2) from the (possible) corresponding inequalities for the polarizations using general weak lower semi-continuity properties. In fact, for any closed half space $H$ with $0 \in H$ and any $u \in W^{1, p}\left(\mathbb{R}^{N}\right)$,

$$
\int_{\mathbb{R}^{N}}\left|\nabla u^{H}\right|^{p} d x=\int_{\mathbb{R}^{N}}|\nabla u|^{p} d x
$$

as well as, for any $W^{s, p}\left(\mathbb{R}^{N}\right)$,

$$
\iint_{\mathbb{R}^{2 N}} \frac{\left|u^{H}(x)-u^{H}(y)\right|^{p}}{|x-y|^{N+p s}} d x d y \leq \iint_{\mathbb{R}^{2 N}} \frac{|u(x)-u(y)|^{p}}{|x-y|^{N+p s}} d x d y,
$$

see $[3,14]$ and references included. For applications of polarization techniques, see $[19,33-35,37,38]$.

More recently, a new class of nonlocal functionals has been involved in the study of topological degree of a map $[6,11,24]$, namely, for $\delta>0$ and $1<p<N$,

$$
I_{\delta}(u):=\iint_{\{|u(y)-u(x)|>\delta\}} \frac{\delta^{p}}{|x-y|^{N+p}} d x d y .
$$

It turns out that this energy also provides a pointwise approximation of $\|\nabla u\|_{p}^{p}$ for $p>1$, precisely

$$
\lim _{\delta \searrow 0} I_{\delta}(u)=\frac{1}{p} K_{N, p} \int_{\mathbb{R}^{N}}|\nabla u|^{p} d x,
$$

where $K_{N, p}$ is given by (1.4), see [7,23] (and also [9,10,12,13,25]). Various properties of Sobolev spaces in terms of $I_{\delta}$ were investigated in [27], for example, it was shown in [27, Theorem 3] that, for $1<p<N$ and for $\delta>0$,

$$
\left(\int_{\{|u|>\lambda \delta\}}|u|^{\frac{N p}{N-p}} d x\right)^{\frac{N-p}{N p}} \leq C\left(I_{\delta}(u)\right)^{1 / p}, \quad \forall u \in L^{p}\left(\mathbb{R}^{N}\right),
$$

for some positive constants $C, \lambda$ depending only on $N$ and $p$.

The first goal of this note is to prove that, however, the nonlocal energy $I_{\delta}$ fails to be decreasing upon polarization. This supports yet again the idea (cf. [26]) that $I_{\delta}$ is a much more delicate approximation of local norm with respect to the other mentioned above. More precisely, we have 
Theorem 1.1. Let $N \geq 1$ and $p \geq 1$. Then there exist $\delta_{0}>0$ and a closed half space $H \subset \mathbb{R}^{N}$ with $0 \in H$ such that for any $0<\delta<\delta_{0}$ there exists a measurable function $u: \mathbb{R}^{N} \rightarrow \mathbb{R}$ such that $I_{\delta}\left(u^{H}\right)>I_{\delta}(u)$.

The proof of Theorem 1.1 is given in Section 2.2. In Section 2.3, we present a proof of (1.2).

The second goal of this note is to prove some facts related to symmetric functions. Precisely:

- (Global compactness and $I_{\delta}$ ). Let $1<p<N,\left(\delta_{n}\right) \rightarrow 0_{+}$, and let $\left\{u_{n}\right\}_{n \in \mathbb{N}}$ be a sequence of radially symmetric decreasing functions. Assume that

$$
\left\{u_{n}\right\}_{n \in \mathbb{N}} \text { is bounded in } L^{p}\left(\mathbb{R}^{N}\right) \text { and }\left\{I_{\delta_{n}}\left(u_{n}\right)\right\}_{n \in \mathbb{N}} \text { is bounded in } \mathbb{R} .
$$

Then $\left\{u_{n}\right\}_{n \in \mathbb{N}}$ is pre-compact in $L^{r}\left(\mathbb{R}^{N}\right)$ for every $p<r<N p /(N-p)$.

- (Decay and integrability of $\left.I_{\delta}\right)$. If $u: \mathbb{R}^{N} \rightarrow \mathbb{R}^{+}$and there exists $\vartheta>0$ with

$$
\int_{0}^{\infty} \frac{I_{\delta}\left(u^{*}\right)^{\vartheta / p}}{\delta} d \delta<\infty
$$

then there exists $C>0$ depending on $u, N, p, \vartheta$ such that $0 \leq u^{*}(x) \leq C|x|^{-(N-p) / p}$.

The proof of these two facts is given in Section 3 (see Theorems 3.2 and 3.4).

\section{Symmetrization inequalities}

\subsection{The defect of decreasingness of $I_{\delta}$}

In the following $H$ will denote a closed half-space of $\mathbb{R}^{N}$ containing the origin. We denote by $\mathcal{H}$ the set of these closed half-spaces. A reflection $\sigma_{H}: \mathbb{R}^{N} \rightarrow \mathbb{R}^{N}$ with respect to $H$ is an isometry such that $\sigma_{H}^{2}=\mathrm{Id}$ and $|x-y|<\left|x-\sigma_{H}(y)\right|$ for all $x, y \in H$. We also set $H^{c}:=\mathbb{R}^{N} \backslash H$. Given $x \in \mathbb{R}^{N}, \sigma_{H}(x)$ will also be denoted by $x^{H}$. The polarization (or two-point rearrangement) of a nonnegative real valued function $u: \mathbb{R}^{N} \rightarrow \mathbb{R}^{+}$ with respect to a given $H$ is defined as

$$
u^{H}(x):=\left\{\begin{array}{ll}
\max \left\{u(x), u\left(\sigma_{H}(x)\right)\right\}, & \text { for } x \in H, \\
\min \left\{u(x), u\left(\sigma_{H}(x)\right)\right\}, & \text { for } x \in H^{c}
\end{array} .\right.
$$

Let us set

$$
\begin{aligned}
& A:=\left\{x \in \operatorname{int}(H): u(x) \leq u\left(x^{H}\right)\right\}, \quad B:=\left\{x \in \operatorname{int}(H): u(x)>u\left(x^{H}\right)\right\}, \\
& C:=\left\{y \in H^{c}: u(y) \geq u\left(y^{H}\right)\right\}, \quad \text { and } \quad D:=\left\{y \in H^{c}: u(y)<u\left(y^{H}\right)\right\} .
\end{aligned}
$$

It is clear that

$$
C=\sigma(A) \quad \text { and } \quad D=\sigma(B)
$$

We have

$$
I_{\delta}=I_{\delta}^{(A \cup C)(A \cup C)}+I_{\delta}^{(B \cup D)(B \cup D)}+I_{\delta}^{(A \cup C)(B \cup D)}+I_{\delta}^{(B \cup D)(A \cup C)},
$$

where, for two measurable subsets $O, P$ of $\mathbb{R}^{N}$, we denote

$$
I_{\delta}^{O P}(u):=\int_{O} \int_{P\{|u(y)-u(x)|>\delta\}} \frac{\delta^{p}}{|x-y|^{N+p}} d x d y .
$$

We claim that

$$
\begin{aligned}
I_{\delta}^{(A \cup C)(A \cup C)}\left(u^{H}\right) & =I_{\delta}^{(A \cup C)(A \cup C)}(u), \\
I_{\delta}^{(B \cup D)(B \cup D)}\left(u^{H}\right) & =I_{\delta}^{(B \cup D)(B \cup D)}(u) .
\end{aligned}
$$


We begin with (2.3). Since

$$
u^{H}(x)=u\left(x^{H}\right) \text { if } x \in A \cup C \quad \text { and } \quad|x-y|=\left|x^{H}-y^{H}\right|,
$$

by a change of variable $x=x^{H}$ and $y=y^{H}$, we obtain

$$
I_{\delta}^{(A \cup C)(A \cup C)}\left(u^{H}\right)=I_{\delta}^{(A \cup C)(A \cup C)}(u),
$$

which is (2.3). We next establish (2.4). This is a direct consequence of the fact

$$
u^{H}(x)=u(x), \quad \text { if } x \in B \cup D .
$$

We next concern about the validity of the inequality:

$$
I_{\delta}^{(A \cup C)(B \cup D)}\left(u^{H}\right) \leq I_{\delta}^{(A \cup C)(B \cup D)}(u) .
$$

By a change of variables, we obtain

$$
I_{\delta}^{(A \cup C)(B \cup D)}(v)=\int_{A} \int_{B}\left(\frac{\mathscr{L}(v, x, y)}{|x-y|^{N+p}}+\frac{\mathscr{L}\left(v, x, y^{H}\right)}{\left|x-y^{H}\right|^{N+p}}+\frac{\mathscr{L}\left(v, x^{H}, y\right)}{\left|x^{H}-y\right|^{N+p}}+\frac{\mathscr{L}\left(v, x^{H}, y^{H}\right)}{\left|x^{H}-y^{H}\right|^{N+p}}\right) d x d y,
$$

where

$$
\mathscr{L}(v, x, y):=\delta^{p} \mathbb{1}_{\{|v(x)-v(y)|>\delta\}}(x, y), \quad x, y \in \mathbb{R}^{N}
$$

We have, for $x \in A$ and $y \in B$,

$$
u^{H}(x)=u\left(x^{H}\right), \quad u^{H}\left(x^{H}\right)=u(x), \quad u^{H}(y)=u(y), \text { and } \quad u^{H}\left(y^{H}\right)=u\left(y^{H}\right) .
$$

It follows that, for $x \in A$ and $y \in B$,

$$
\frac{\mathscr{L}\left(u^{H}, x, y\right)}{|x-y|^{N+p}}=\frac{\mathscr{L}\left(u, x^{H}, y\right)}{|x-y|^{N+p}}, \quad \frac{\mathscr{L}\left(u^{H}, x, y^{H}\right)}{\left|x-y^{H}\right|^{N+p}}=\frac{\mathscr{L}\left(u, x^{H}, y^{H}\right)}{\left|x-y^{H}\right|^{N+p}}
$$

and

$$
\frac{\mathscr{L}\left(u^{H}, x^{H}, y\right)}{\left|x^{H}-y\right|^{N+p}}=\frac{\mathscr{L}(u, x, y)}{\left|x^{H}-y\right|^{N+p}}, \quad \frac{\mathscr{L}\left(u^{H}, x^{H}, y^{H}\right)}{\left|x^{H}-y^{H}\right|^{N+p}}=\frac{\mathscr{L}\left(u, x, y^{H}\right)}{|x-y|^{N+p}} .
$$

Setting

$$
\mathscr{D}_{\delta}^{H}(u, x, y):=\mathscr{L}\left(u, x^{H}, y\right)+\mathscr{L}\left(u, x, y^{H}\right)-\mathscr{L}(u, x, y)-\mathscr{L}\left(u, x^{H}, y^{H}\right),
$$

we derive that inequality $(2.5)$ is equivalent to

$$
\mathfrak{d}_{\delta}^{H}(u):=\int_{A} \int_{B} \mathscr{D}_{\delta}^{H}(u, x, y)\left(\frac{1}{|x-y|^{N+p}}-\frac{1}{\left|x^{H}-y\right|^{N+p}}\right) d x d y \leq 0 .
$$

On the other hand, in general, concrete examples show that the inequality $\mathscr{D}_{u}(x, y)$ fails, so that it is expected that $I_{\delta}\left(u^{H}\right)-I_{\delta}(u)$ can be positive for some $H, u$ and $\delta>0$, in which case the quantity $\mathfrak{d}_{\delta}^{H}(u)$ provides a measure of the defect of decreasingness. 
Remark 2.1 (Vanishing Defect). For any closed half space $H \subset \mathbb{R}^{N}$ with $0 \in H$ there holds, for $p>1$,

$$
\lim _{\delta \searrow 0} \mathfrak{d}_{\delta}^{H}(u)=0, \quad \text { for all } u \in W^{1, p}\left(\mathbb{R}^{N}\right) .
$$

In fact, we know that $u^{H} \in W^{1, p}\left(\mathbb{R}^{N}\right)$ also and $\left\|\nabla u^{H}\right\|_{L^{p}\left(\mathbb{R}^{N}\right)}=\|\nabla u\|_{L^{p}\left(\mathbb{R}^{N}\right)}$ from formula (1.5). Furthermore, from formulas (2.2)-(2.5), we infer

$$
\mathfrak{d}_{\delta}^{H}(u)=\frac{1}{2} I_{\delta}\left(u^{H}\right)-\frac{1}{2} I_{\delta}(u), \quad \text { for all } \delta>0 .
$$

Then, from equality (1.7), we conclude

$$
\lim _{\delta \searrow 0} \mathfrak{d}_{\delta}^{H}(u)=\frac{1}{2} \lim _{\delta \searrow 0} I_{\delta}\left(u^{H}\right)-\frac{1}{2} \lim _{\delta \searrow 0} I_{\delta}(u)=\frac{K_{N, p}}{2 p}\left(\left\|\nabla u^{H}\right\|_{L^{p}\left(\mathbb{R}^{N}\right)}^{p}-\|\nabla u\|_{L^{p}\left(\mathbb{R}^{N}\right)}^{p}\right)=0,
$$

proving the assertion.

\subsection{Proof of Theorem 1.1}

We first deal with the case $N=1$. Here is a counterexample to $(2.6)$ with $p \in[1,+\infty)$. Fix $\varepsilon \in(0,1 / 8)$ and let $u: \mathbb{R} \rightarrow \mathbb{R}$ be defined by

$$
u(x)=\left\{\begin{array}{cl}
\delta & \text { for } x \in(-2,-1], \\
\text { linear } & \text { for } x \in[-1,-1+\delta], \\
-2 \varepsilon \delta & \text { for } x \in[-1+\delta, 0), \\
-\varepsilon \delta & \text { for } x \in(0,1), \\
\delta-\varepsilon \delta & \text { for } x \in(1,2), \\
0 & \text { for } x \notin(-2,2) .
\end{array}\right.
$$

Let $H=[0, \infty)$ and $\sigma$ be the standard reflection. It is clear that $u^{H}$ satisfies

$$
u^{H}(x)=\left\{\begin{array}{cl}
\delta-\varepsilon \delta & \text { for } x \in(-2,-1] \\
-2 \varepsilon \delta & \text { for } x \in[-1+\delta, 0) \\
-\varepsilon \delta & \text { for } x \in(0,1-\delta), \\
\delta & \text { for } x \in(1,2) \\
0 & \text { for } x \notin(-2,2)
\end{array}\right.
$$

We derive from (2.7) and (2.8) that

$$
\begin{aligned}
I_{\delta}\left(u^{H}\right)-I_{\delta}(u) \geq & \int_{0}^{1-\delta} \int_{1}^{2} \frac{\delta^{p}}{|x-y|^{p+1}} d x d y \\
& -2 \int_{-1}^{-1+\delta} \int_{-2}^{0} \frac{\delta^{p}}{|x-y|^{p+1}} d x d y-2 \int_{-1}^{0} \int_{1}^{2} \frac{\delta^{p}}{|x-y|^{p+1}} d x d y .
\end{aligned}
$$

A straightforward computation yields, for small $\delta$ and $\varepsilon$,

$$
\int_{0}^{1-\delta} \int_{1}^{2} \frac{\delta^{p}}{|x-y|^{p+1}} d x d y \simeq\left\{\begin{array}{cl}
\delta|\ln \delta| & \text { if } p=1 \\
\delta & \text { if } p>1,
\end{array} \int_{-1}^{-1+\delta} \int_{-2}^{0} \frac{\delta^{p}}{|x-y|^{p+1}} d x d y \simeq \varepsilon \delta\right.
$$

and

$$
\int_{-1}^{-0} \int_{1}^{2} \frac{\delta^{p}}{|x-y|^{p+1}} d x d y \simeq \delta^{p}
$$


We obtain that, for small positive $\delta$ and $\varepsilon$, we have $I_{\delta}\left(u^{H}\right)>I_{\delta}(u)$.

This example can be modified to obtain similar conclusion in the case $\{0\} \in H$ by considering the function $u(\cdot+c)$ for some $c>0$ where $u$ is given above. In the above example, the function $u$ is not non-negative. However, this point can be handled by considering the function given by $u(x)+2 \varepsilon \delta$ if $|x|<3$ and 0 otherwise.

We next consider the case $N \geq 2$. Set

$$
H=[0,+\infty) \times \mathbb{R}^{N-1}
$$

and define $U: \mathbb{R}^{N} \rightarrow \mathbb{R}$ as follows, for $\left(x_{1}, x^{\prime}\right) \in \mathbb{R} \times \mathbb{R}^{N-1}$,

$$
U\left(x_{1}, x^{\prime}\right)=\left\{\begin{array}{cl}
u\left(x_{1}\right)-\delta / 2 & \text { if }|x|_{\infty} \leq 2, \\
0 & \text { otherwise }
\end{array}\right.
$$

where $u$ is given in (2.7). One can check that

$$
I_{\delta}\left(U^{H}\right)-I_{\delta}(U) \simeq I_{\delta}\left(u^{H}\right)-I_{\delta}(u)>0 .
$$

In the above example, the function $U$ is not non-negative and $H$ does not contain the origin. However, this point can be handled similarly as in the case $N=1$.

The following question remains open:

Open problem 2.2. Let $N \geq 1$. It is true that $I_{\delta}\left(u^{*}\right) \leq I_{\delta}(u)$ for any measurable $u: \mathbb{R}^{N} \rightarrow \mathbb{R}^{+}$and $\delta>0$ ?

\subsection{Riesz two-point inequality}

Let $u \in L_{\text {loc }}^{1}\left(\mathbb{R}^{N}\right)$, let $H$ be a closed half-space of $\mathbb{R}^{N}$ and let $G$ be a Young function, i.e. $G:[0,+\infty) \rightarrow \mathbb{R}$, $G(0)=0, G$ is non-decreasing, and $G$ is convex, and let $w$ be a non-negative, non-increasing radial function. The above notations allow to prove the classical inequality

$$
\iint_{\mathbb{R}^{2 N}} G\left(\left|u^{H}(x)-u^{H}(y)\right|\right) w(|x-y|) d x d y \leq \iint_{\mathbb{R}^{2 N}} G(|u(x)-u(y)|) w(|x-y|) d x d y,
$$

where $G(u, x, y)$ means $G(|u(x)-u(y)|)$. In fact, set

$$
\mathscr{D}^{H}(u, x, y):=G\left(u, x^{H}, y\right)+G\left(u, x, y^{H}\right)-G(u, x, y)-G\left(u, x^{H}, y^{H}\right),
$$

and define

$$
\mathfrak{d}^{H}(u):=\iint_{\mathbb{R}^{2 N}} G\left(\left|u^{H}(x)-u^{H}(y)\right|\right) w(|x-y|) d x d y-\iint_{\mathbb{R}^{2 N}} G(|u(x)-u(y)|) w(|x-y|) d x d y .
$$

As in (2.6), we have

$$
\mathfrak{d}^{H}(u)=\int_{A} \int_{B} \mathscr{D}^{H}(x, y ; u)\left(w(|x-y|)-w\left(\left|x^{H}-y\right|\right)\right) d x d y .
$$

We claim that

$$
\mathscr{D}^{H}(u, x, y) \leq 0
$$

for each $x \in A$ and $y \in B$. Assuming this, we then immediately get inequality (1.6) since $w$ is non-decreasing. We now prove (2.10). Observe that if $a \leq b$ and $c \leq d$ then

$$
|a-c|+|b-d| \leq|d-a|+|b-c|
$$


and

$$
\max \{|a-c|,|b-d|\} \leq \max \{|d-a|,|b-c|\}
$$

It follows that, for $x \in A$ and $y \in B$,

$$
\left|u(x)-u\left(y^{H}\right)\right|+\left|u\left(x^{H}\right)-u(y)\right| \leq|u(y)-u(x)|+\left|u\left(x^{H}\right)-u\left(y^{H}\right)\right|
$$

and

$$
\max \left\{\left|u(x)-u\left(y^{H}\right)\right|,\left|u\left(x^{H}\right)-u(y)\right|\right\} \leq \max \left\{|u(y)-u(x)|,\left|u\left(x^{H}\right)-u\left(y^{H}\right)\right|\right\} .
$$

Assertion (2.10) follows from the properties of Young's functions of $G$.

As a consequence of $(2.9)$, one has

$$
\left|u^{H}\right|_{W^{s, p}\left(\mathbb{R}^{N}\right)} \leq|u|_{W^{s, p}\left(\mathbb{R}^{N}\right)},
$$

for $s \in(0,1)$ and $p>1$. It follows that, for $u \in W^{s, p}\left(\mathbb{R}^{N}\right)$ with $u>0$,

$$
\left|u^{*}\right|_{W^{s, p}\left(\mathbb{R}^{N}\right)} \leq|u|_{W^{s, p}\left(\mathbb{R}^{N}\right)},
$$

where $u^{*}$ denotes the spherical symmetric rearrangement of $u$. By the BBM formula (1.3), one reaches the Polya-Szegö inequality.

Remark 2.3. Recall that $\mathcal{H}$ is the set of half-spaces which contain the origin. One can endow $\mathcal{H}$ with a metric that ensures that $H_{n} \rightarrow H$ if there exists a sequence of isometries $i_{n}: \mathbb{R}^{N} \rightarrow \mathbb{R}^{N}$ such that $H_{n}=i_{n}(H)$ and $i_{n}$ converges to the identity as $n \rightarrow+\infty$, moreover, $\mathcal{H}$ is separable with respect to this metric. Let $\left\{H_{n}\right\}_{n \in \mathbb{N}}$ be a dense set in $\mathcal{H}$. For any $u \in L^{p}\left(\mathbb{R}^{N}\right)$, let $\left\{u_{n}\right\}_{n \in \mathbb{N}}$ be the sequence defined by

$$
u_{0}:=u \quad \text { and } \quad u_{n+1}:=u_{n}^{H_{1} \cdots H_{n+1}} \text { for } n \geq 0 .
$$

Assume that

$$
\sup _{n \in \mathbb{N}} I_{\delta_{n}}\left(u_{n}\right)<+\infty, \quad \text { for some }\left\{\delta_{n}\right\} \rightarrow 0 .
$$

Then $u^{*} \in W^{1, p}\left(\mathbb{R}^{N}\right)(p>1)$, where $u^{*}$ denotes the Schwarz symmetrization of $u$. In fact, by [38, Theorem 1], we have $u_{n} \rightarrow u^{*}$ strongly in $L^{p}\left(\mathbb{R}^{N}\right)$ as $n \rightarrow \infty$. Then, the assertion follows by the Gamma-convergence result in [26, Theorem 2]. If in addition, for all $\delta>0$,

$$
\iint_{\{|u(y)-u(x)|>\delta\}}|x-y|^{-N-p} d x d y=\iint_{\left\{\left|u^{*}(y)-u^{*}(x)\right|>\delta\right\}}|x-y|^{-N-p} d x d y,
$$

then $u$ is radially symmetric about some point $x_{0} \in \mathbb{R}^{N}$ provided that $\mathcal{L}^{N}\left(\left\{\nabla u^{*}=0\right\}\right)=0$. This follows by the Brothers-Ziemer result [15, Theorem 1.1] jointly with [23, Theorem 2].

\section{Radially decreasing functions and $I_{\delta}$}

For every measurable function $u: \mathbb{R}^{N} \rightarrow \mathbb{R}$ we define its distribution function

$$
\mu_{u}(t)=|\{x:|u(x)|>t\}|, \quad t>0 .
$$


Let $0<q<\infty$ and $0<\vartheta<\infty$, the Lorentz space $L^{q, \vartheta}\left(\mathbb{R}^{N}\right)$ (cf. [21,22,28]) is defined by

$$
L^{q, \vartheta}\left(\mathbb{R}^{N}\right):=\left\{u: \mathbb{R}^{N} \rightarrow \mathbb{R}: \int_{0}^{\infty} t^{\vartheta-1} \mu_{u}(t)^{\vartheta / q} d t<\infty\right\} .
$$

In the limit case $\vartheta=\infty$, this is defined by

$$
L^{q, \infty}\left(\mathbb{R}^{N}\right):=\left\{u: \mathbb{R}^{N} \rightarrow \mathbb{R}: \sup _{t>0} t \mu_{u}(t)^{1 / q}<\infty\right\} .
$$

We recall from [8, Lemma 2.9] the following

Lemma 3.1. Let $0<\vartheta \leq \infty$ and $0<q<\infty$. Let $u \in L^{q, \vartheta}\left(\mathbb{R}^{N}\right)$ be a non-negative and radially symmetric decreasing function. Then

$$
\begin{aligned}
& 0 \leq u(x) \leq\left(\vartheta \omega_{N}^{-\frac{\vartheta}{q}} \int_{0}^{\infty} t^{\vartheta-1} \mu_{u}(t)^{\frac{\vartheta}{q}} d t\right)^{\frac{1}{\vartheta}}|x|^{-\frac{N}{q}}, \quad \text { if } \vartheta<\infty, \\
& 0 \leq u(x) \leq\left(\omega_{N}^{-\frac{1}{q}} \sup _{t>0} t \mu_{u}(t)^{\frac{1}{q}}\right)|x|^{-\frac{N}{q}}, \quad \text { if } \vartheta=\infty .
\end{aligned}
$$

The next proposition shows that the measure of the superlevels of a nonnegative function $u$ is controlled by a quantity involving $I_{\delta}\left(u^{*}\right)$. Set $p^{*}:=N p /(N-p)$ for $1 \leq p<N$.

Theorem 3.2. Let $1<p<N$ and $u: \mathbb{R}^{N} \rightarrow \mathbb{R}$ be a non-negative function. Then there exist two positive constants $C$ and $\lambda$ depending only on $N$ and $p$ such that

$$
\left|\left\{x \in \mathbb{R}^{N}: u(x)>\lambda \delta\right\}\right| \leq C \delta^{-\frac{N p}{N-p}} \min \left\{I_{\delta}\left(u^{*}\right)^{\frac{N}{N-p}}, I_{\delta}\left(u^{*}\right)^{\frac{N}{N-p}}\right\},
$$

where $u^{*}$ is the Schwarz symmetric rearrangement of $u$. Assume that there exists $\vartheta>0$ such that

$$
\int_{0}^{\infty} \frac{I_{\delta}(u)^{\frac{\vartheta}{p}}}{\delta} d \delta<\infty \quad \text { or } \quad \int_{0}^{\infty} \frac{I_{\delta}\left(u^{*}\right)^{\frac{\vartheta}{p}}}{\delta} d \delta<\infty .
$$

Then $u, u^{*} \in L^{p^{*}, \vartheta}\left(\mathbb{R}^{N}\right)$ and there exists a positive constant $C$ depending on $u, N, p, \vartheta$ such that

$$
0 \leq u^{*}(x) \leq C|x|^{-\frac{N-p}{p}} .
$$

Proof. By the definition of $u^{*}$ we have that $\mu_{u}(t)=\mu_{u^{*}}(t)$ for $t>0$. Then, by applying inequality (1.8) to $u^{*}$, we have, for all $\delta>0$,

$$
\lambda \delta \mu_{u}(\lambda \delta)^{\frac{N-p}{N p}}=\lambda \delta \mu_{u^{*}}(\lambda \delta)^{\frac{N-p}{N p}} \leq\left(\int_{\left\{\left|u^{*}\right|>\lambda \delta\right\}}\left|u^{*}\right|^{\frac{N p}{N-p}} d x\right)^{\frac{N-p}{N p}} \leq C I_{\delta}\left(u^{*}\right)^{\frac{1}{p}}
$$

and

$$
\lambda \delta \mu_{u^{*}}(\lambda \delta)^{\frac{N-p}{N p}}=\lambda \delta \mu_{u}(\lambda \delta)^{\frac{N-p}{N p}} \leq\left(\int_{\left\{\left|u^{*}\right|>\lambda \delta\right\}}|u|^{\frac{N p}{N-p}} d x\right)^{\frac{N-p}{N p}} \leq C I_{\delta}(u)^{\frac{1}{p}},
$$

which implies (3.1). By virtue of (3.2), it follows from (3.1) that

$$
\int_{0}^{\infty} \delta^{\vartheta-1} \mu_{u}(\delta)^{\frac{\vartheta}{p^{*}}} d \delta<\infty \quad \text { and } \quad \int_{0}^{\infty} \delta^{\vartheta-1} \mu_{u^{*}}(\delta)^{\frac{\vartheta}{p^{*}}} d \delta<\infty
$$

which yields $u, u^{*} \in L^{p^{*}, \vartheta}\left(\mathbb{R}^{N}\right)$ and the final assertions follow from Lemma 3.1.

Remark 3.3. Since $L^{q, q}\left(\mathbb{R}^{N}\right)=L^{q}\left(\mathbb{R}^{N}\right)$, it follows that $u, u^{*} \in L^{p^{*}}\left(\mathbb{R}^{N}\right)$ if

$$
\int_{0}^{\infty} \frac{I_{\delta}(u)^{N /(N-p)}}{\delta} d \delta<\infty \quad \text { or } \quad \int_{0}^{\infty} \frac{I_{\delta}\left(u^{*}\right)^{N /(N-p)}}{\delta} d \delta<\infty .
$$


Concerning the compactness related to $I_{\delta}$, the following result was shown in [27, Theorem 2]. Let $p>1$, $\left(\delta_{n}\right) \rightarrow 0_{+}$and $\left(u_{n}\right) \subset L^{p}\left(\mathbb{R}^{N}\right)$. Assume that

$$
\left\{u_{n}\right\}_{n \in \mathbb{N}} \text { is bounded in } L^{p}\left(\mathbb{R}^{N}\right) \text { and }\left\{I_{\delta_{n}}\left(u_{n}\right)\right\}_{n \in \mathbb{N}} \text { is bounded. }
$$

Then

$$
\left\{u_{n}\right\}_{n \in \mathbb{N}} \text { is pre-compact in } L_{\text {loc }}^{p}\left(\mathbb{R}^{N}\right) .
$$

In this paper, we prove the following global compactness result:

Theorem 3.4. Let $1<p<N,\left(\delta_{n}\right) \rightarrow 0_{+}$, and let $\left\{u_{n}\right\}_{n \in \mathbb{N}}$ be a sequence of radially symmetric decreasing functions. Assume that

$$
\left\{u_{n}\right\}_{n \in \mathbb{N}} \text { is bounded in } L^{p}\left(\mathbb{R}^{N}\right) \text { and }\left\{I_{\delta_{n}}\left(u_{n}\right)\right\}_{n \in \mathbb{N}} \text { is bounded. }
$$

Then $\left\{u_{n}\right\}_{n \in \mathbb{N}}$ is pre-compact in $L^{r}\left(\mathbb{R}^{N}\right)$ for every $p<r<N p /(N-p)$.

Proof. From (1.8), (3.3), and (3.4), we derive that

$$
\left(u_{n}\right) \text { is pre-compact in } L_{\text {loc }}^{r}\left(\mathbb{R}^{N}\right) .
$$

Since $\left(u_{n}\right)$ is decreasing and $\left(u_{n}\right)$ is bounded in $L^{p}\left(\mathbb{R}^{N}\right)$, for any $\delta>0$ there exists $R_{\delta}$ such that, for all $n$,

$$
\left|u_{n}(x)\right| \leq \delta \text { for }|x|>R_{\delta} .
$$

Fix $\varepsilon>0$. By (3.5), there exists a finite subset $J$ of $\mathbb{N}$ such that

$$
\left\{u_{n} \in L^{r}\left(B_{R_{\varepsilon}}\right) ; n \in \mathbb{N}\right\} \subset \bigcup_{j \in J}\left\{u \in L^{r}\left(B_{R_{\varepsilon}}\right):\left\|u-u_{j}\right\|_{L^{r}\left(B_{R_{\varepsilon}}\right)}<\varepsilon\right\},
$$

where $B_{R}$ denotes the open ball centered at the origin and of radius $R$ in $\mathbb{R}^{N}$ for $R>0$. On the other hand, by (3.6), we have

$$
\left\|u_{n}\right\|_{L^{r}\left(\mathbb{R}^{N} \backslash B_{R_{\varepsilon}}\right)} \leq \varepsilon^{(r-p) / r}\left\|u_{n}\right\|_{L^{p}\left(\mathbb{R}^{N} \backslash B_{R_{\varepsilon}}\right)}^{p / r} \leq C \varepsilon^{1-p / r},
$$

since $\left(u_{n}\right)$ is bounded in $L^{p}\left(\mathbb{R}^{N}\right)$. A combination of (3.7) and (3.8) yields, for $\varepsilon$ small enough such that $\varepsilon<C \varepsilon^{1-p / r}$,

$$
\left\{u_{n} \in L^{r}\left(\mathbb{R}^{N}\right) ; n \in \mathbb{N}\right\} \subset \bigcup_{j \in J}\left\{u \in L^{r}\left(\mathbb{R}^{N}\right) ;\left\|u-u_{j}\right\|_{L^{r}\left(\mathbb{R}^{N}\right)}<3 C \varepsilon^{1-p / r}\right\},
$$

where $C$ is the constant in (3.8). Since (3.9) holds for small $\varepsilon$ and $1-r / p>0$, it follows that $\left(u_{n}\right)$ is pre-compact in $L^{r}\left(\mathbb{R}^{N}\right)$.

Remark 3.5. Let $N \geq 1$ and $p \geq N$. It was shown in [27, Theorem 1] that if $u \in L^{p}\left(\mathbb{R}^{N}\right)$ and $I_{\delta}(u)<+\infty$ for some $\delta>0$ then $u \in B M O_{\mathrm{loc}}\left(\mathbb{R}^{N}\right)$ where $B M O$ denotes the space of functions of bounded mean oscillation. By the same proof, under the same assumptions on $\left\{u_{n}\right\}$, one obtains the compactness result for $L^{r}\left(\mathbb{R}^{N}\right)$ for $p<r<p^{*}$ with $p^{*}=\infty$.

As a consequence of Theorem 3.4, we have the following 
Corollary 3.6. Let $1<p<N$ and let $\left\{u_{n}\right\}_{n \in \mathbb{N}}$ be a nonnegative sequence of functions bounded in $L^{p}\left(\mathbb{R}^{N}\right)$ such that

$$
\liminf _{n \rightarrow \infty} I_{\delta_{n}}\left(u_{n}^{*}\right)<\infty,
$$

for some sequence $\left\{\delta_{n}\right\}_{n \in \mathbb{N}} \rightarrow 0_{+}$. Then, up to a subsequence, $\left\{u_{n}^{*}\right\}_{n \in \mathbb{N}}$ converges strongly in $L^{r}\left(\mathbb{R}^{N}\right)$ to a radial decreasing function, for any $p<r<N p /(N-p)$.

Proof. Since the sequence $\left\{u_{n}\right\}_{n \in \mathbb{N}}$ is bounded in $L^{p}\left(\mathbb{R}^{N}\right)$, it follows that sequence $\left\{u_{n}^{*}\right\}_{n \in \mathbb{N}}$ is also bounded in $L^{p}\left(\mathbb{R}^{N}\right)$ by Cavalieri's principle. Then, by Theorem 3.4, it follows that $u_{n}^{*} \rightarrow v$ in $L^{r}\left(\mathbb{R}^{N}\right)$ strongly for any $p<r<N p /(N-p)$.

\section{An open problem for Riesz fractional gradients}

Recently, a notion of fractional gradients (more precisely distributional Riesz fractional gradients) has been introduced in the literature by Shieh and Spector in the papers [31,32], where several basic properties of local Sobolev spaces (e.g. Sobolev, Morrey, Hardy, Trudinger inequalities) are proven to extend to fractional spaces defined through this new notion.

More precisely, the fractional gradient $D^{s} u(x)$ at a point $x \in \mathbb{R}^{N}$ is defined for locally Lipschitz compactly supported functions $u: \mathbb{R}^{N} \rightarrow \mathbb{R}$, for any $s \in(0,1)$, by

$$
D^{s} u(x):=c_{N, s} \int_{\mathbb{R}^{N}} \frac{u(x)-u(y)}{|x-y|^{N+s}} \frac{x-y}{|x-y|} d y, \quad \text { for } x \in \mathbb{R}^{N},
$$

for a suitable positive constant $c_{N, s}$ depending on $N$ and $s$. This is reminiscent of the classical scalar notion of $\frac{s}{2}$-fractional laplacian

$$
(-\Delta)^{s / 2} u(x)=C_{N, s} \int_{\mathbb{R}^{N}} \frac{u(x)-u(y)}{|x-y|^{N+s}} d y, \quad \text { for } x \in \mathbb{R}^{N},
$$

for a suitable normalization constant $C_{N, s}$ depending on $N$ and $s$. Notice also that $[31,32]$

$$
D^{s} u=I_{1-s} * D u, \quad u \in C_{c}^{\infty}\left(\mathbb{R}^{N}\right), \quad I_{1-s}(x):=\frac{\gamma(N, s)}{|x|^{N+s-1}} .
$$

According to [31,32], one can define, for $p>1$ and $s \in(0,1)$, the space

$$
L^{s, p}\left(\mathbb{R}^{N}\right):=\overline{C_{c}^{\infty}\left(\mathbb{R}^{N}\right)}\|\cdot\|_{L^{p}}+\left\|D^{s} \cdot\right\|_{L^{p}} .
$$

We now formulate a related open problem.

Open problem 4.1. Let $p>1, s \in(0,1)$ and $u \in L^{s, p}\left(\mathbb{R}^{N}\right)$ with $u \geq 0$. Prove or disprove that $D^{s} u^{*} \in L^{s, p}\left(\mathbb{R}^{N}\right)$ and the inequality holds

$$
\int_{\mathbb{R}^{N}}\left|D^{s} u^{*}\right|^{p} d x \leq \int_{\mathbb{R}^{N}}\left|D^{s} u\right|^{p} d x
$$

In general the inequality

$$
\int_{\mathbb{R}^{N}}\left|D^{s}\right| u||^{p} d x \leq \int_{\mathbb{R}^{N}}\left|D^{s} u\right|^{p} d x .
$$

for all $u \in L^{s, p}\left(\mathbb{R}^{N}\right)$ is not expected to hold. In particular, one cannot obtain inequality (4.1) for signchanging functions. The solution of the above open problem would be very useful in connection with compact 
injections for radially symmetric functions of $L^{s, p}\left(\mathbb{R}^{N}\right)$. In fact, assume $N \geq 2$. Notice that, since for any $\varepsilon \in(0, s)$ the injection

$$
L^{s, p}\left(\mathbb{R}^{N}\right) \hookrightarrow W^{s-\varepsilon, p}\left(\mathbb{R}^{N}\right),
$$

is continuous (cf. [31, (g) of Theorem 2.2]) and the injection

$$
W_{\text {rad }}^{s-\varepsilon, p}\left(\mathbb{R}^{N}\right) \hookrightarrow L^{q}\left(\mathbb{R}^{N}\right),
$$

is compact (cf. [20, Theorem II.1]) for all $p<q<p_{s-\varepsilon}^{*}$ it follows that the injection

$$
L_{\mathrm{rad}}^{s, p}\left(\mathbb{R}^{N}\right) \hookrightarrow L^{q}\left(\mathbb{R}^{N}\right),
$$

is compact for $p<q<N p /(N-s p)$. In particular, nonnegative minimizing sequences for

$$
u \mapsto \int_{\mathbb{R}^{N}}\left|D^{s} u\right|^{p} d x
$$

could be replaced by new minimizing sequences which are radially symmetric decreasing and strongly converging in $L^{q}\left(\mathbb{R}^{N}\right)$ for any $p<q<N p /(N-s p)$.

Remark 4.2. Aiming to prove (4.1), with no loss of generality one may assume $u \in C_{c}^{\infty}\left(\mathbb{R}^{N}\right)$. Let $H$ be an arbitrary closed given half-space with $0 \in H$. Define the function $\mathfrak{J}(u): \mathbb{R}^{N} \rightarrow \mathbb{R}$,

$$
\mathfrak{J}(u)(x):=\left|\int_{\mathbb{R}^{N}} \frac{u(x)-u(y)}{|x-y|^{N+s}} \frac{x-y}{|x-y|} d y\right|^{p}, \quad \text { for all } x \in \mathbb{R}^{N} .
$$

Then $\mathfrak{J}(u) \in L^{1}\left(\mathbb{R}^{N}\right)$. Setting $v(x):=u\left(x^{H}\right)$ and $w(x):=u^{H}\left(x^{H}\right)$ for all $x \in \mathbb{R}^{N}$, we have

$$
u^{H}(x)=v(x)+(u(x)-v(x))^{+}, \quad w(x)=u(x)-(u(x)-v(x))^{+}, \quad \text { for } x \in H .
$$

Writing $x^{H}=x_{0}+R x$ where $R$ is a rotation, a change of variable yields

$$
\begin{aligned}
\mathfrak{J}(u)\left(x^{H}\right) & =\left|\int_{\mathbb{R}^{N}} \frac{u\left(x^{H}\right)-u(y)}{\left|x^{H}-y\right|^{N+s}} \frac{x^{H}-y}{\left|x^{H}-y\right|} d y\right|^{p} \\
& =\left|\int_{\mathbb{R}^{N}} \frac{u\left(x^{H}\right)-u\left(y^{H}\right)}{\left|x^{H}-y^{H}\right|^{N+s}} \frac{x^{H}-y^{H}}{\left|x^{H}-y^{H}\right|} d y\right|^{p} \\
& =\left|R \int_{\mathbb{R}^{N}} \frac{v(x)-v(y)}{|x-y|^{N+s}} \frac{x-y}{|x-y|} d y\right|^{p}=\mathfrak{J}(v)(x),
\end{aligned}
$$

and, analogously, $\mathfrak{J}\left(u^{H}\right)\left(x^{H}\right)=\mathfrak{J}(w)(x)$. In turn, we conclude that

$$
\int_{\mathbb{R}^{N}} \mathfrak{J}(u) d x=\int_{H} \mathfrak{J}(u) d x+\int_{H} \mathfrak{J}(v) d x, \quad \int_{\mathbb{R}^{N}} \mathfrak{J}\left(u^{H}\right) d x=\int_{H} \mathfrak{J}\left(u^{H}\right) d x+\int_{H} \mathfrak{J}(w) d x .
$$

If one was be able to prove that

$$
\mathfrak{J}\left(u^{H}\right)(x)+\mathfrak{J}(w)(x) \leq \mathfrak{J}(u)(x)+\mathfrak{J}(v)(x), \quad \text { for all } x \in H,
$$

then (4.1) would follow by standard approximations. In the local case (4.3) follows immediately in light of (4.2), while for $\mathfrak{J}$, which is a nonlocal function, the situation is rather unclear.

If instead one finds $u$ and $H$ such that (4.3) holds with opposite inequality, then the Riesz gradients would already fail the basic polarization inequality. 


\section{Acknowledgments}

The authors would like to warmly thank Daniel Spector for providing some useful remarks about the content of his works

\section{References}

[1] F.J. Almgren, E.H. Lieb, Symmetric decreasing rearrangement is sometimes continuous, J. Amer. Math. Soc. 2 (1989) 683-773.

[2] T. Aubin, Problémes isopérimétriques et espaces de sobolev, J. Differential Geom. 11 (1976) 573-598.

[3] A. Baernstein, A unified approach to symmetrization, in: A. Alvino et al. (Ed.), Partial Differential Equations of Elliptic Type, in: Symposia Matematica, vol. 35, Cambridge University Press, 1995, pp. 47-91.

[4] J. Bourgain, H. Brezis, P. Mironescu, Another look at Sobolev spaces, in: J.L. Menaldi, E. Rofman, A. Sulem (Eds.) Optimal Control and Partial Differential Equations, in: A Volume in Honor of Professor Alain Bensoussan's 60th Birthday, IOS Press, Amsterdam, 2001, pp. 439-455.

[5] J. Bourgain, H. Brezis, P. Mironescu, Limiting embedding theorems for $W^{s, p}$ when $s \uparrow 1$ and applications, J. Anal. Math. 87 (2002) 77-101.

[6] J. Bourgain, H. Brezis, H.-M. Nguyen, A new estimate for the topological degree, C. R. Math. Acad. Sci. Paris 340 (2005) $787-791$.

[7] J. Bourgain, H.-M. Nguyen, A new characterization of Sobolev spaces, C. R. Math. Acad. Sci. Paris 343 (2006) 75-80.

[8] L. Brasco, S. Mosconi, M. Squassina, Optimal decay of extremals for the fractional Sobolev inequality, Calc. Var. Partial Differential Equations 55 (2016) 55:23.

[9] H. Brezis, How to recognize constant functions. Connections with Sobolev spaces, Russian Math. Surveys 57 (2002) 693-708.

[10] H. Brezis, H.-M. Nguyen, Non-local functionals related to the total variation and connections with Image Processing, preprint. http://arxiv.org/abs/1608.08204.

[11] H. Brezis, H.-M. Nguyen, On the distributional Jacobian of maps from $S^{N}$ into $S^{N}$ in fractional Sobolev and Hölder spaces, Ann. of Math 173 (2011) 1141-1183.

[12] H. Brezis, H.-M. Nguyen, The BBM formula revisited, Atti Accad. Naz. Lincei Rend. Lincei Mat. Appl. 27 (2016) 515-533.

[13] H. Brezis, H.-M. Nguyen, Two subtle convex nonlocal approximations of the BV-norm, Nonlinear Anal. 137 (2016) $222-245$.

[14] F. Brock, Y. Solynin, An approach to symmetrization via polarization, Trans. Amer. Math. Soc. 352 (2000) 1759-1796.

[15] J.E. Brothers, W.P. Ziemer, Minimal rearrangements of Sobolev functions, J. Reine Angew. Math. 384 (1988) 153-179.

[16] V.N. Dubinin, Transformation of functions and the Dirichlet principle, Mat. Zametki 38 (1985) 49-55.

[17] V.N. Dubinin, Transformation of condensers in space, Dokl. Akad. Nauk SSSR 296 (1987) 18-20.

[18] V.N. Dubinin, Transformations of condensers in an $n$-dimensional space, Zap. Nauchn. Sem. Leningrad. Otdel. Mat. Inst. Steklov. (LOMI) 196: Modul. Funktsii Kvadrat. Formy. 2 (1991) 41-60, 173.

[19] R. Filippucci, P. Pucci, C. Varga, Symmetry and multiple solutions for certain quasilinear elliptic equations, Adv. Differential Equations 20 (2015) 601-634.

[20] P.L. Lions, Symétrie et compacité dans les espaces de Sobolev, J. Funct. Anal. 49 (1982) 315-334.

[21] G. Lorentz, Some new function spaces, Ann. of Math. 51 (1950) 37-55.

[22] G. Lorentz, On the theory of spaces A, Pacific J. Math. 1 (1951) 411-429.

[23] H.-M. Nguyen, Some new characterizations of Sobolev spaces, J. Funct. Anal. 237 (2006) 689-720.

[24] H.-M. Nguyen, Optimal constant in a new estimate for the degree, J. Anal. Math. 101 (2007) 367-395.

[25] H.-M. Nguyen, Further characterizations of Sobolev spaces, J. Eur. Math. Soc. 10 (2008) 191-229.

[26] H.-M. Nguyen, $\Gamma$-convergence, Sobolev norms, and BV functions, Duke Math. J. 157 (2011) $495-533$.

[27] H.-M. Nguyen, Some inequalities related to Sobolev norms, Calc. Var. Partial Differential Equations 41 (2011) $483-509$.

[28] L. Pick, A. Kufner, O. John, S. Fucik, Function Spaces. Vol. 1, second ed., in: De Gruyter Series in Nonlinear Analysis and Applications, vol. 14, Walter de Gruyter, Berlin, 2013.

[29] G. Polya, G. Szegö, Isoperimetric Inequalities in Mathematical Physics, in: Annals of Mathematics Studies, Princeton University Press, Princeton, NJ, 1945.

[30] G. Polya, G. Szegö, Inequalities for the capacity of a condenser, Amer. J. Math. 67 (1945) 1-32.

[31] T.-T. Shieh, D.E. Spector, On a new class of fractional partial differential equations, Adv. Calc. Var. 8 (2015) 321-336.

[32] T.-T. Shieh, D.E. Spector, On a new class of fractional partial differential equations II, Adv. Calc. Var. (2017) in press.

[33] M. Squassina, On a result by boccardo-ferone-fusco-orsina, Rend. Lincei Mat. Appl. 22 (2011a) 505-511.

[34] M. Squassina, Radial symmetry of minimax critical points for nonsmooth functionals, Commun. Contemp. Math. 13 (2011) $487-508$.

[35] M. Squassina, Symmetry in variational principles and applications, J. Lond. Math. Soc. 85 (2012) $323-348$.

[36] G. Talenti, Best constant in Sobolev inequality, Ann. Mat. Pura Appl. 110 (1976) 353-372.

[37] J. Van Schaftingen, Symmetrization and minimax principles, Commun. Contemp. Math. 7 (2005) $463-481$.

[38] J. Van Schaftingen, Explicit approximation of the symmetric rearrangement by polarizations, Arch. Math. 93 (2009) $181-190$. 